\title{
EFICIÊNCIA DE DUAS VARIAÇÕES ESTRUTURAIS DO MÉTODO DE AMOSTRAGEM DE ÁREA FIXA EM PLANTAÇÕES DE Pinus taeda
}

\author{
João Paulo Druszcz ${ }^{1}$, Nelson Yoshihiro Nakajima ${ }^{2}$, Sylvio Péllico Netto ${ }^{2}$, \\ Sebastião do Amaral Machado ${ }^{2}$, Nelson Carlos Rosot, Rogério Bamberg ${ }^{4}$ \\ ${ }^{1}$ Eng. Florestal, Doutorando em Engenharia Florestal, UFPR, Curitiba, PR, Brasil - jpaulorz@ yahoo.com.br \\ ${ }^{2}$ Eng. Florestal, Dr., Depto. de Ciências Florestais, UFPR, Curitiba, PR, Brasil - nelson.nakajima@ufpr.br; \\ sylviopelliconetto@gmail.com; samachado@ufpr.br; ncrosot@ufpr.br \\ ${ }^{4}$ Eng. Florestal, Mestrando em Engenharia Florestal, UFPR, Curitiba, PR, Brasil - rogeriobamber@yahoo.com.br \\ Recebido para publicação: 20/11/2012 - Aceito para publicação: 12/09/2013
}

\begin{abstract}
Resumo
Este estudo foi conduzido em três diferentes condições de plantações de Pinus taeda L., sem desbastes, com 10, 9 e 7 anos de idade, 2.000 árvores por hectare e diferentes inclinações no terreno, localizados no Estado do Paraná. O objetivo foi avaliar a eficiência do inventário florestal, utilizandose a amostragem de área fixa com a estrutura de parcela circular (PC) e conglomerado em cruz (CC), através da análise do comportamento quanto às precisões e eficiências relativas nas estimativas das seguintes variáveis: diâmetro médio $(\mathrm{cm})$, número de árvores por ha, área basal $\left(\mathrm{m}^{2} / \mathrm{ha}\right)$ e volume total $\left(\mathrm{m}^{3} / \mathrm{ha}\right)$. Para isso, utilizou-se o delineamento em blocos casualizados com 40 unidades amostrais para a PC e 10 unidades para o CC, sendo este composto por 4 subunidades circulares. Concluiu-se que, para as variáveis diâmetro médio $(\mathrm{cm})$, número de árvores $(\mathrm{N} / \mathrm{ha})$, área basal $\left(\mathrm{m}^{2} / \mathrm{ha}\right)$ e volume total $\left(\mathrm{m}^{3} / \mathrm{ha}\right)$, indica-se a utilização do método de área fixa com PC, tendo em vista a maior eficiência no levantamento dessas variáveis.

Palavras-chave: Eficiência relativa; precisão; reflorestamento.
\end{abstract}

\begin{abstract}
Structural efficiency of two variations of method of sampling of fixed area in plantations of Pinus taeda. This study was carried out in three different stands of Pinus taeda L., unthinned and aged 10, 9 and 7 years. The stands have 2.000 trees per hectare and are located in Parana State. The aim was to evaluate the efficiency of inventories using circular plot (PC) and cross cluster (CC) by analyses of behavior towards accuracies and relative efficiencies for estimation of the following variables: average diameter at breast height, number of trees, basal area and total volume per hectare. For this study, it was taken a sample of 40 units to the PC structure and 10 units for the CC structure, which is composed of four circular subunits, and it was used the randomized block design. It was concluded that for the variable diameter $(\mathrm{cm})$, number of trees (ha), basal area $\left(\mathrm{m}^{2} / \mathrm{ha}\right)$ and total volume $\left(\mathrm{m}^{3} / \mathrm{ha}\right)$ it is indicated the use of PC, since its greater efficiency in the survey of these variables.

Keywords: Relative efficiency; accuracy; reforestation.
\end{abstract}

\section{INTRODUÇÃO}

O inventário florestal é a ferramenta básica para a obtenção fidedigna de informações necessárias ao planejamento da produção e manejo florestal em empreendimentos de base florestal e, apesar de inovações tecnológicas, como, por exemplo, a tecnologia LiDAR (light detection and ranging), as técnicas de amostragem em campo para a coleta de informações quantitativas e qualitativas dos estoques florestais, por enquanto, dificilmente serão abolidas. Porém, para a obtenção dessas informações, há um custo, que aumenta quanto mais detalhes, nível de precisão e velocidade são requeridos.

Freese (1962) propõe incluir o custo como um indicador para comparar tamanhos de unidades amostrais, tendo ele importante participação no contexto de avaliação da eficiência das unidades amostrais de diferentes tamanhos, podendo-se ainda usar a combinação dos erros amostrais ou dos coeficientes de variação com os respectivos custos de amostragem. 
Avery e Burkhart (1983) afirmam que a melhor estrutura de amostragem de um dado problema de estimativa é aquela que estabelece a precisão desejada pelo menor custo. Para eles, isso é feito com o produto entre o quadrado do erro padrão e o tempo (custo $=$ tempo).

Segundo os pesquisadores Péllico Netto e Brena (1997), em qualquer aplicação de amostragem, a precisão e custo são duas variáveis intimamente interligadas, e a especificação de uma implica automaticamente a determinação da outra. Se o objetivo for comparar vários tamanhos simultaneamente, pode-se calcular o inverso dos produtos dos quadrados dos coeficientes de variação pelos respectivos custos e compará-los entre si.

Nakajima (1997) afirma que, para se compararem diferentes métodos de amostragem, como o de área fixa, utilizando-se diferentes formas nas unidades amostrais, com os de área variável, como os métodos de Bitterlich e Strand, deve-se "transformar" os métodos para uma mesma base de comparação, como a padronização do número de árvores por unidade amostral. Segundo Nakajima (1998), a eficiência dos diferentes métodos de amostragem (tipo, forma e tamanho das parcelas) varia de acordo com o tipo florestal (florestas plantadas ou naturais), com as condições da floresta e com a topografia da região de estudo, entre outros aspectos. Ainda conforme Nakajima (1997), a adequação do método de amostragem para as condições da topografia e da floresta possibilitará um aumento na precisão, para um mesmo esforço de amostragem, nas estimativas das variáveis de interesse e, consequentemente, uma redução do tempo de execução, o que refletirá na redução de custos.

Sparcks et al. (2002) compararam estimativas de número de árvores em três plantios no sudeste de Oklahoma (EUA), usando parcelas circulares e quadradas de tamanho fixo e de tamanho variável. Concluíram que todas as parcelas foram eficientes quanto ao tempo de medição, mas as de área fixa forneceram estimativas mais precisas do número de árvores, independentemente das suas dimensões.

Mauricio et al. (2005), em seus estudos em plantios de Pinus caribaea Barr. et Golf. na parte oriental da Venezuela, concluíram que o método de inventário com parcelas de área fixa é levemente mais preciso que o de área variável, principalmente quanto à estimativa da variável número de árvores. Os mesmos autores afirmam que a menor precisão do método de área variável é compensada pela maior velocidade de execução e economia nos custos de inventário.

Druszcz et al. (2010), no estudo da comparação entre o método de amostragem de Bitterlich e o de área fixa com parcela circular, na obtenção das variáveis diâmetro médio, número de árvores, área basal e volume, por hectare, conduzido em plantios de Pinus taeda L. no estado do Paraná, concluíram que, para as condições pesquisadas, o método de área fixa com parcela circular foi mais preciso e eficiente na obtenção das variáveis diâmetro médio e número de árvores por hectare; e, para a obtenção das variáveis área basal e volume, por hectare, o método de Bitterlich.

Nakajima et al. (2011) estudaram a precisão do método de amostragem em linha e com parcela circular concêntrica em plantios de Pinus elliottii Engelm., no sul do estado de São Paulo, para as variáveis diâmetro médio, número de árvores, área basal e volume, por hectare, e concluíram que, para as condições estudadas, o método de amostragem em linha foi superior.

Druszcz et al. (2012), no estudo da precisão e eficiência dos métodos de amostragem de área variável de Bitterlich e de área fixa com conglomerado em cruz, conduzido em plantios de Pinus taeda $\mathrm{L}$. localizados no estado do Paraná, verificaram que, para as condições pesquisadas, o método de Bitterlich foi mais preciso e eficiente na obtenção das variáveis diâmetro médio, área basal e volume, por hectare, e o método de área fixa com conglomerado em cruz foi mais preciso e eficiente na obtenção da variável número de árvores. Druszcz et al. (2012), em estudo da eficiência em inventário florestal, utilizando a amostragem ponto de Bitterlich e conglomerado em linha, conduzido em plantios de Pinus taeda L. localizados no estado do Paraná, concluíram que, para as condições pesquisadas, o método de área fixa com conglomerado em linha foi mais preciso e eficiente na obtenção das variáveis diâmetro médio e número de árvores, por hectare, e para as variáveis área basal e volume, por hectare, o método de Bitterlich foi mais preciso e eficiente.

Dessa forma, o objetivo deste estudo foi comparar a eficiência de duas variações estruturais do método de área fixa, ou seja, parcela circular (PC) versus conglomerado em cruz (CC), em inventário florestal para as estimativas correntes das variáveis diâmetro médio $(\mathrm{cm})$, número de árvores $(\mathrm{N} / \mathrm{ha})$, área basal $\left(\mathrm{m}^{2} / \mathrm{ha}\right)$ e volume total $\left(\mathrm{m}^{3} / \mathrm{ha}\right)$, em plantações de Pinus taeda L., através de suas precisões e eficiências relativas. Nesse contexto, parte-se da hipótese de que não existem diferenças significativas estatisticamente entre a eficiência em inventários florestais realizados por ambas as variações estruturais, isto é, quaisquer diferenças observadas são devidas exclusivamente aos fatores não controlados ou ao acaso. 


\section{MATERIAL E MÉTODOS}

\section{Área de estudo}

A área deste estudo está localizada na região de Ponta Grossa, estado do Paraná. As coordenadas geográficas são $24^{\circ} 04^{\prime} 44^{\prime \prime}$ de latitude sul e $50^{\circ} 05^{\prime} 49^{\prime \prime}$ de longitude oeste. O clima é classificado como Cfb de Köppen, ou seja, clima subtropical úmido, mesotérmico, com verões frescos, geadas severas demasiado frequentes e sem estação seca, sendo a precipitação anual média acima de $1.400 \mathrm{~mm}$, com chuvas bem distribuídas durante o ano. A temperatura média anual nos meses mais frios é inferior a $18{ }^{\circ} \mathrm{C}$, enquanto que a média anual dos meses mais quentes é superior a $22^{\circ} \mathrm{C}$ (BOGNOLA et al., 2002).

Os talhões utilizados neste estudo são de Pinus taeda L., sem desbastes, com espaçamentos de $2,00 \mathrm{~m}$ por $2,50 \mathrm{~m}$. As unidades amostrais foram alocadas em 3 diferentes talhões, denominados de condições 1,2 e 3 . A condição 1 tem área de efetivo plantio igual a 52,10 ha, idade de 10 anos e topografia com $4^{\circ}$ de inclinação média; a condição 2 tem área de efetivo plantio igual a 41,52 ha, idade de 7 anos e topografia com $5,5^{\circ}$ de inclinação média; e a condição 3 tem área de efetivo plantio igual a 32,86 ha, idade de 9 anos e topografia com $6,5^{\circ}$ de inclinação média. As três condições totalizam uma área de 126,48 ha.

\section{Delineamento experimental utilizado}

O delineamento experimental utilizado foi o de blocos casualizados com repetições, isto é, cada condição topográfica/etária foi considerada um bloco e dentro de cada um deles foram sorteados ao acaso 40 pontos amostrais para o método de área fixa com parcela circular (PC) e 10 pontos amostrais para o método de área fixa com conglomerado em cruz (CC). Para o controle das condições experimentais, esses pontos amostrais foram utilizados para os dois métodos.

\section{Equações volumétrica e hipsométrica utilizadas}

Os volumes e alturas totais das árvores foram estimados por meio das seguintes equações utilizadas para cada uma das condições, as quais foram geradas com dados deste levantamento.

\section{Condição 1}

Equação de volume total, com casca, para árvore individual (Modelo Schumacher-Hall):

$$
\operatorname{Ln} \hat{V}_{i}=-10,60677+1,95794 \cdot \ln D A P_{i}+1,19020 \cdot \ln H_{i}
$$

Equação hipsométrica para altura total (Modelo de Curtis):

$$
\operatorname{Ln} \hat{H}_{i}=3,48436+21,30533 .\left(1 / D A P_{i}\right)+107,04890 .\left(1 / D A P_{i}^{2}\right)
$$

\section{Condição 2}

Equação de volume total, com casca, para árvore individual (Modelo Schumacher-Hall):

$$
\operatorname{Ln} \hat{V}_{i}=-9,67886+1,96111 \cdot \ln D A P_{i}+0,82583 \cdot \ln H_{i}
$$

Equação hipsométrica para altura total (Modelo de Ogawa):

$$
1 / \hat{H}_{i}=0,052045+0,80003.1 / \mathrm{DAP} P_{i}
$$

\section{Condição 3}

Equação de volume total, com casca, para árvore individual (Modelo Schumacher-Hall):

$$
\operatorname{Ln} \hat{V}_{i}=-9,58424+1,90806 \cdot \ln D A P_{i}+0,86390 \cdot \ln H_{i}
$$

Equação hipsométrica para altura total (Modelo de Trorey):

$$
\hat{H}_{i}=9,81650-0,06225 . D A P_{i}+0,00778 . D A P_{i}^{2} \text { ) }
$$




\section{Método de área fixa com parcela circular (PC)}

Segundo Sanquetta et al. (2009), as unidades circulares ainda são menos utilizadas no Brasil que as retangulares e quadradas, porém seu uso vem sendo cada vez mais frequente em inventários florestais. $\mathrm{Na}$ condição1, o raio da unidade amostral foi de $6,75 \mathrm{~m}$, que corresponde a uma área de $143,14 \mathrm{~m}^{2}$ ou 0,0143 ha. Para a condição 2, o raio da unidade amostral foi de 5,65 m, que corresponde a uma área de $100,29 \mathrm{~m}^{2}$ ou $0,01 \mathrm{ha}$, e, para a condição 3, o raio da unidade amostral foi de 5,60 m, que corresponde a uma área de $98,52 \mathrm{~m}^{2}$ ou 0,0099 ha. As fórmulas usadas para as estimativas das variáveis foram sugeridas por Nishizawa (1972).

$$
\begin{array}{lr}
d m=\frac{\sum_{i=1}^{n} d_{i}}{n} & N=\frac{n}{a} \\
G=\frac{\sum_{i=1}^{n} g_{i}}{a} & V=\frac{\sum_{i=1}^{n} v_{i}}{a}
\end{array}
$$

Em que: $d m=$ diâmetro médio $(\mathrm{cm})$;

$N=$ número de árvores por hectare;

$G=$ área basal por hectare $\left(\mathrm{m}^{2} / \mathrm{ha}\right)$;

$V=$ volume por hectare $\left(\mathrm{m}^{3} / \mathrm{ha}\right)$;

$n=$ número de árvores selecionadas por ponto de amostragem;

$d_{i}=$ diâmetro da árvore "i" selecionada $(\mathrm{cm})$;

$g_{i}=$ área transversal da árvore " $\mathrm{i}$ " selecionada $\left(\mathrm{m}^{2}\right)$;

$v_{i}=$ volume da árvore "i $\mathrm{i}$ " selecionada $\left(\mathrm{m}^{3}\right)$.

$a=$ área da parcela circular (ha).

\section{Método de área fixa com conglomerado em cruz (CC)}

As unidades de amostra em conglomerados são casos especiais de unidades amostrais de área fixa circulares, quadradas ou retangulares, em que se reúne um grupo de subunidades para compor uma unidade principal denominada conglomerado ou cluster, em inglês (SANQUETTA et al., 2009). Neste estudo, foram utilizadas subunidades amostrais com diferentes áreas, ou seja, para cada condição foi determinado um raio. Na condição 1 , o raio da subunidade amostral foi de $6,75 \mathrm{~m}$, que corresponde a uma área de $143,14 \mathrm{~m}^{2}$ ou 0,0143 ha. Para a condição 2 , o raio da subunidade amostral foi de 5,65 m, que corresponde a uma área de $100,29 \mathrm{~m}^{2}$ ou 0,01 ha, e para a condição 3 , o raio da subunidade amostral foi de 5,60 m, que corresponde a uma área de $98,52 \mathrm{~m}^{2}$ ou 0,0099 ha. A distância entre os centros das subunidades foi calculada da seguinte forma: uma distância fixa de $10 \mathrm{~m}$ entre bordas das subunidades, mais os raios que, para as condições acima mencionadas, geraram uma distância entre os centros das subunidades de $23,50 \mathrm{~m}, 21,30 \mathrm{~m}$ e $21,20 \mathrm{~m}$.

As fórmulas usadas para as estimativas das variáveis foram sugeridas por Queiroz (1998), modificadas por Druszcz (2008).

$$
\begin{array}{cc}
\overline{d_{i j}}=\frac{\sum_{k=1}^{n_{i k}} d_{i j k}}{n_{i j k}} & d m=\frac{\sum_{i=1}^{n} \sum_{j=1}^{M} \overline{d_{i j}}}{n M} \\
N A=\left(\frac{\sum_{i=1}^{n} \sum_{j=1}^{M} n_{i j}}{n \cdot M}\right) \frac{1}{s} & G=\frac{\sum_{i=1}^{n} \sum_{j=1}^{M}\left(\sum_{k=1}^{n i j k} g i j k\right)}{n M} \frac{1}{s}
\end{array}
$$




$$
V=\frac{\sum_{i=1}^{n} \sum_{j=1}^{M}\left(\sum_{k=1}^{n i j k} v i j k\right)}{n M} \frac{1}{S}
$$

Em que: $n=$ número de unidades primárias ou conglomerados amostrados;

$M$ = número de subunidades que compõem cada conglomerado;

$d m=$ diâmetro médio do conglomerado $(\mathrm{cm})$;

$N A$ = número de árvores por hectare;

$G=$ área basal por hectare $\left(\mathrm{m}^{2} / \mathrm{ha}\right)$;

$V=$ volume por hectare $\left(\mathrm{m}^{3} / \mathrm{ha}\right)$;

$\overline{d_{i j}}=$ diâmetro médio das árvores selecionadas na subunidade “j” do conglomerado “i”;

$n_{i j k}=$ número de árvores " $\mathrm{k}$ " selecionada na subunidade " $\mathrm{j}$ " no conglomerado "i";

$g_{i j k}=$ área basal da árvore "k" selecionada na subunidade "j" no conglomerado "i";

$v_{i j k}=$ volume da árvore " $\mathrm{k}$ " selecionada na subunidade "j" no conglomerado “i”;

$s$ = área da subunidade circular (ha).

\section{Intensidade amostral ou repetições}

No presente estudo, cada estrutura foi conduzida em três diferentes condições topográficas/etárias, com uma intensidade amostral de 40 unidades para cada condição para a estrutura com PC e 10 unidades para a estrutura com CC. O processo de amostragem adotado para sorteio das unidades amostrais na área do estudo foi o inteiramente ao acaso.

\section{Método da eficiência relativa (ER)}

Para o cálculo de eficiência relativa, foram consideradas todas as variáveis estimadas em ambas as estruturas do método de área fixa.

Freese (1962) considera os tempos médios de mensuração em cada uma das estruturas e os coeficientes de variação obtidos. No caso do presente trabalho, a estrutura que apresentar maior valor de eficiência relativa é a mais eficiente, calculada pela seguinte fórmula:

Em que: $E R=$ eficiência relativa;

$$
E R=\frac{1}{T_{i} C V^{2}}
$$

$$
\begin{aligned}
& T_{i}=\text { tempo de medição da unidade amostral " } i \text { "; } \\
& C V=\text { coeficiente de variação. }
\end{aligned}
$$

Sendo assim, pode-se, com eficiência relativa, comparar as duas estruturas do método de área fixa e mostrar qual delas teve melhor desempenho, atendendo ao limite de erro estipulado.

\section{Método de medição}

Para estimativa das variáveis diâmetro médio $(\mathrm{cm})$, número de árvores por $(\mathrm{N} / \mathrm{ha})$, área basal $\left(\mathrm{m}^{2} / \mathrm{ha}\right)$ e volume total $\left(\mathrm{m}^{3} / \mathrm{ha}\right)$, foram coletados dados de diâmetro à altura do peito (DAP) e também foram medidas, com o auxílio do hipsômetro de Blume-Leiss, as alturas de 120 árvores, ao acaso, presentes nas unidades amostrais. Adicionalmente, foi mensurado o tempo de implantação e a medição das parcelas, com o auxílio de um cronômetro digital.

\section{Instrumentos e materiais utilizados na medição}

As medições das árvores inclusas nas parcelas foram feitas pelo método direto, tanto para a PC quanto para o CC, com o uso dos seguintes instrumentos e materiais: estaca para marcação do ponto 
amostral, trena para medição do raio da parcela e/ou raio das subunidades, fita métrica graduada em milímetros para medição das circunferências à altura do peito (CAPs) e material para anotação.

\section{Análises estatísticas}

Análise estatística para a estrutura com parcela circular (PC)

Para a amostragem utilizando a PC, as análises estatísticas foram realizadas por meio das seguintes fórmulas, conforme Péllico Netto e Brena (1997).

$$
\begin{gathered}
\bar{x}=\frac{\sum_{i=1}^{n}\left(x_{i}\right)}{n} \\
s_{x}=\sqrt{s_{x}^{2}} \\
c v=\frac{s_{x}}{x} 100
\end{gathered}
$$

$$
\begin{gathered}
s_{x}^{2}=\frac{\sum_{i=1}^{n}\left(x_{i}-\bar{x}\right)^{2}}{n-1} \\
s_{\bar{x}}= \pm \frac{s_{x}}{\sqrt{n}} \\
e r= \pm \frac{t s_{-}}{\bar{x}} 100
\end{gathered}
$$

$$
i c=\bar{x} \pm t s_{\bar{x}}
$$

Em que: $\bar{x}=$ média da variável $\mathrm{x}$;

$x_{i}=$ valor de cada variável $\mathrm{x}$;

$n=$ número de unidades amostrais;

$s^{2}=$ variância;

$s$ = desvio padrão;

$S_{\bar{x}}=$ erro padrão;

er $=$ erro de amostragem em porcentagem;

$t=$ valor tabelar de Student $\left(\mathrm{t}_{(39 ; 0.05)}=2,023\right)$;

$i c=$ intervalo de confiança.

Para esta estrutura, a população foi considerada infinita, sendo o erro máximo admissível de $10 \%$ e a probabilidade baseada em $95 \%$.

Análise estatística para a estrutura com conglomerado em cruz (CC)

Para a amostragem utilizando o CC, as análises estatísticas foram realizadas por meio das seguintes fórmulas, sugeridas por Péllico Netto e Brena (1997):

$$
\begin{array}{cc}
\bar{x}=\frac{\sum_{i=1}^{n} \sum_{j=1}^{M} X_{i j}}{n M} & \bar{x}_{i}=\sum_{j=1}^{M} \frac{X_{i j}}{M} \\
s_{x}^{2}=s_{e}^{2}+s_{d}^{2} & s_{d}^{2}=M Q_{\text {dentro }}=\frac{\sum_{i=1}^{n} \sum_{j=1}^{M}\left(X i_{j}-\bar{x}_{i}\right)^{2}}{n(M-1)} \\
s_{e}^{2}=\frac{M Q_{\text {entre }}-M Q_{\text {dentro }}}{M} & \text { em que } \quad M Q_{\text {entre }}=\frac{\sum_{i=1}^{n} M\left(\bar{x}_{i}-\bar{x}\right)^{2}}{n-1}
\end{array}
$$




$$
\begin{gathered}
r=\frac{s_{e}^{2}}{s_{e}^{2}+s_{d}^{2}} \\
s=\sqrt{s_{e}^{2}+s_{d}^{2}} \\
s_{-}=\sqrt{s_{\frac{x}{x}}^{2}}
\end{gathered}
$$

$$
\begin{gathered}
s_{\frac{x}{2}}^{2}=\frac{s_{x}^{2}}{n M}[1+r(M-1)] \\
c v=\frac{s}{x} 100 \\
e r= \pm \frac{t s_{-}}{\bar{x}} 100
\end{gathered}
$$

$i c= \pm t s_{\bar{x}}$

Em que: $\bar{x}=$ média da população por subunidade;

$x_{i}=$ média das subunidades por conglomerado;

$n$ = número de conglomerados amostrados;

$s_{x}^{2}=$ variância da população por subunidade;

$s_{e}^{2}=$ variância entre conglomerados;

$s_{d}^{2}=$ variância dentro dos conglomerados;

$Q M_{\text {entre }}=$ quadrado médio entre os conglomerados;

$Q M_{\text {dentro }}=$ quadrado médio entre as subunidades dentro dos conglomerados;

$r$ = coeficiente de correlação intraconglomerados;

$s_{\frac{1}{x}}^{2}=$ variância da média;

$S=$ desvio padrão;

$S_{-}=$erro padrão;

$c v=$ coeficiente de variação;

er = erro de amostragem em porcentagem;

$t=$ valor tabelar de Student $\left(\mathrm{t}_{(09 ; 0.05)}=2,262\right)$;

ic $=$ intervalo de confiança.

Para esta estrutura, a população foi considerada infinita, sendo o erro máximo admissível de $10 \%$, com $95 \%$ de probabilidade.

\section{RESULTADOS E DISCUSSÃO}

\section{Número de árvores amostradas por unidade amostral ou parcela}

Foram medidas as CAPs de 4.830 árvores em 150 unidades amostrais instaladas em uma área experimental de 126,48 ha. Na tabela 1, é apresentado o número de árvores amostradas em cada unidade amostral ou parcela, por condição e estrutura.

O tamanho da amostra das duas estruturas foi o mesmo, porém observa-se que o número de árvores amostrado pela $\mathrm{PC}$ foi $1,7 \%$ maior que pelo $\mathrm{CC}$, em média.

\section{Estimativa das variáveis pelas estruturas $\mathrm{PC}$ e CC}

$\mathrm{Na}$ tabela 2 está apresentado o resumo das estimativas dos valores das variáveis DAP médio $(\mathrm{cm})$, número de árvores $(\mathrm{N} / \mathrm{ha})$, área basal $\left(\mathrm{m}^{2} / \mathrm{ha}\right)$ e volume total $\left(\mathrm{m}^{3} / \mathrm{ha}\right)$ para cada condição. 
Tabela 1. Número de árvores por unidade amostral para as três condições.

Table 1. Number of trees by sampling unit for the three conditions.

\begin{tabular}{|c|c|c|c|c|c|c|}
\hline \multirow{2}{*}{$\begin{array}{l}\text { Unidade } \\
\text { amostral }\end{array}$} & \multicolumn{2}{|c|}{ Condição 1} & \multicolumn{2}{|c|}{ Condição 2} & \multicolumn{2}{|c|}{ Condição 3} \\
\hline & PC & $\mathrm{CC}$ & $\mathbf{P C}$ & $\mathrm{CC}$ & PC & $\mathrm{CC}$ \\
\hline 1 & 23 & 93 & 18 & 75 & 20 & 70 \\
\hline 2 & 22 & 95 & 20 & 77 & 18 & 77 \\
\hline 3 & 22 & 85 & 19 & 71 & 19 & 72 \\
\hline 4 & 22 & 92 & 20 & 81 & 18 & 78 \\
\hline 5 & 22 & 94 & 20 & 73 & 18 & 66 \\
\hline 6 & 24 & 86 & 21 & 78 & 18 & 70 \\
\hline 7 & 22 & 92 & 20 & 79 & 17 & 69 \\
\hline 8 & 24 & 90 & 18 & 73 & 18 & 75 \\
\hline 9 & 24 & 102 & 19 & 67 & 18 & 81 \\
\hline 10 & 23 & 87 & 19 & 74 & 18 & 73 \\
\hline 11 & 23 & & 19 & & 16 & \\
\hline 12 & 25 & & 19 & & 19 & \\
\hline 13 & 21 & & 16 & & 18 & \\
\hline 14 & 20 & & 21 & & 20 & \\
\hline 15 & 22 & & 21 & & 18 & \\
\hline 16 & 24 & & 20 & & 20 & \\
\hline 17 & 22 & & 17 & & 17 & \\
\hline 18 & 24 & & 23 & & 18 & \\
\hline 19 & 23 & & 19 & & 16 & \\
\hline 20 & 24 & & 20 & & 18 & \\
\hline 21 & 25 & & 19 & & 19 & \\
\hline 22 & 25 & & 18 & & 19 & \\
\hline 23 & 23 & & 19 & & 17 & \\
\hline 24 & 23 & & 23 & & 18 & \\
\hline 25 & 26 & & 19 & & 19 & \\
\hline 26 & 24 & & 18 & & 19 & \\
\hline 27 & 24 & & 18 & & 18 & \\
\hline 28 & 25 & & 16 & & 19 & \\
\hline 29 & 22 & & 16 & & 18 & \\
\hline 30 & 24 & & 19 & & 19 & \\
\hline 31 & 21 & & 22 & & 18 & \\
\hline 32 & 23 & & 20 & & 19 & \\
\hline 33 & 25 & & 21 & & 18 & \\
\hline 34 & 25 & & 21 & & 20 & \\
\hline 35 & 22 & & 19 & & 20 & \\
\hline 36 & 23 & & 18 & & 20 & \\
\hline 37 & 25 & & 19 & & 18 & \\
\hline 38 & 22 & & 19 & & 17 & \\
\hline 39 & 23 & & 22 & & 19 & \\
\hline 40 & 21 & & 17 & & 20 & \\
\hline Soma & 927 & 916 & 772 & 748 & 736 & 731 \\
\hline Média & 23 & 23 & 19 & 19 & 18 & 18 \\
\hline
\end{tabular}


Tabela 2. Estimativa das variáveis DAP médio $(\mathrm{cm})$, número de árvores $(\mathrm{N} / \mathrm{ha})$, área basal $\left(\mathrm{m}^{2} / \mathrm{ha}\right)$ e volume total $\left(\mathrm{m}^{3} / \mathrm{ha}\right)$ para as três condições.

Table 2. Estimate of the variables mean DBH, number of trees, basal area and volume by ha for the three conditions.

\begin{tabular}{lcccccc}
\hline \multirow{2}{*}{ Variáveis } & \multicolumn{2}{c}{ Condição 1 } & \multicolumn{2}{c}{ Condição 2 } & \multicolumn{2}{c}{ Condição 3 } \\
\cline { 2 - 7 } & PC & CC & PC & CC & PC & CC \\
\hline DAP médio $(\mathrm{cm})$ & 16,6 & 16,5 & 16,0 & 16,0 & 15,3 & 15,1 \\
Número de árvores/ha & 1619 & 1600 & 1924 & 1865 & 1868 & 1855 \\
Área basal/ha $\left(\mathrm{m}^{2}\right)$ & 36,94 & 36,24 & 40,15 & 38,90 & 35,90 & 34,76 \\
Volume/ha $\left(\mathrm{m}^{3}\right)$ & 237,24 & 231,87 & 191,96 & 185,78 & 192,56 & 185,88 \\
\hline
\end{tabular}

Análises estatísticas das variáveis estimadas pelas duas estruturas

Os resumos das análises estatísticas das variáveis DAP médio $(\mathrm{cm})$, número de árvores $(\mathrm{N} / \mathrm{ha})$, área basal ( $\left.\mathrm{m}^{2} / \mathrm{ha}\right)$ e volume total $\left(\mathrm{m}^{3} / \mathrm{ha}\right)$, estão apresentados na tabela 3 .

Tabela 3. Análises estatísticas para o DAP médio $(\mathrm{cm})$, número de árvores $(\mathrm{N} / \mathrm{ha})$, área basal $\left(\mathrm{m}^{2} / \mathrm{ha}\right)$ e volume total $\left(\mathrm{m}^{3} / \mathrm{ha}\right)$ estimado para as três condições.

Table 3. Statistical analyses for estimating mean DBH, number of trees, basal area e volume for the three conditions.

\begin{tabular}{|c|c|c|c|c|c|c|}
\hline \multirow{2}{*}{ DAP médio (cm) } & \multicolumn{2}{|c|}{ Condição 1} & \multicolumn{2}{|c|}{ Condição 2} & \multicolumn{2}{|c|}{ Condição 3} \\
\hline & $\mathbf{P C}$ & $\mathrm{CC}$ & $\mathbf{P C}$ & CC & PC & CC \\
\hline Desvio padrão $(\mathrm{cm})$ & $\pm 0,89$ & $\pm 1,02$ & $\pm 0,96$ & $\pm 0,93$ & $\pm 0,87$ & $\pm 0,89$ \\
\hline Erro padrão (cm) & $\pm 0,14$ & $\pm 0,26$ & $\pm 0,15$ & $\pm 0,24$ & $\pm 0,14$ & $\pm 0,22$ \\
\hline Coeficiente de variação (\%) & 5,34 & 6,17 & 5,98 & 5,83 & 5,65 & 5,91 \\
\hline Erro de amostragem (\%) & $\pm 1,71$ & $\pm 3,20$ & $\pm 1,91$ & $\pm 3,08$ & $\pm 1,81$ & $\pm 2,89$ \\
\hline Intervalo de confiança $(\mathrm{cm})$ & $\pm 0,28$ & $\pm 0,53$ & $\pm 0,31$ & $\pm 0,49$ & $\pm 0,28$ & $\pm 0,44$ \\
\hline \multirow{2}{*}{ Número de árvores/ha } & \multicolumn{2}{|c|}{ Condição 1} & \multicolumn{2}{|c|}{ Condição 2} & \multicolumn{2}{|c|}{ Condição 3} \\
\hline & $\mathbf{P C}$ & $\mathrm{CC}$ & $\mathbf{P C}$ & CC & PC & CC \\
\hline Desvio padrão (N/ha) & $\pm 97,38$ & $\pm 133,12$ & $\pm 170,78$ & $\pm 182,96$ & $\pm 107,32$ & $\pm 203,85$ \\
\hline Erro padrão (N/ha) & $\pm 15,40$ & $\pm 27,70$ & $\pm 27,00$ & $\pm 32,57$ & $\pm 16,97$ & $\pm 37,15$ \\
\hline Coeficiente de variação (\%) & 6,01 & 8,32 & 8,87 & 9,81 & 5,75 & 10,99 \\
\hline Erro de amostragem $(\%)$ & $\pm 1,92$ & $\pm 3,50$ & $\pm 2,84$ & $\pm 3,53$ & $\pm 1,84$ & $\pm 4,05$ \\
\hline Intervalo de confiança (N/ha) & $\pm 31,14$ & $\pm 56,03$ & $\pm 54,62$ & $\pm 65,87$ & $\pm 34,32$ & $\pm 75,14$ \\
\hline \multirow{2}{*}{ Área basal $\left(\mathbf{m}^{2} / \mathrm{ha}\right)$} & \multicolumn{2}{|c|}{ Condição 1} & \multicolumn{2}{|c|}{ Condição 2} & \multicolumn{2}{|c|}{ Condição 3} \\
\hline & $\mathbf{P C}$ & $\mathrm{CC}$ & $\mathbf{P C}$ & $\mathrm{CC}$ & PC & $\mathrm{CC}$ \\
\hline Desvio padrão $\left(\mathrm{m}^{2} / \mathrm{ha}\right)$ & $\pm 3,54$ & $\pm 5,24$ & $\pm 5,02$ & $\pm 4,22$ & $\pm 4,05$ & $\pm 4,03$ \\
\hline Erro padrão (m²/ha) & $\pm 0,56$ & $\pm 1,36$ & $\pm 0,79$ & $\pm 0,76$ & $\pm 0,64$ & $\pm 0,74$ \\
\hline Coeficiente de variação (\%) & 9,60 & 14,47 & 12,51 & 10,84 & 11,27 & 11,59 \\
\hline Erro de amostragem (\%) & $\pm \mathbf{3 , 0 7}$ & $\pm 7,57$ & $\pm 4,00$ & $\pm 3,94$ & $\pm 3,61$ & $\pm 4,28$ \\
\hline Intervalo de confiança $\left(\mathrm{m}^{2} / \mathrm{ha}\right)$ & $\pm 1,13$ & $\pm 2,74$ & $\pm 1,61$ & $\pm 1,53$ & $\pm 1,29$ & $\pm 1,49$ \\
\hline \multirow{2}{*}{ Volume total (m3/ha) } & \multicolumn{2}{|c|}{ Condição 1} & \multicolumn{2}{|c|}{ Condição 2} & \multicolumn{2}{|c|}{ Condição 3} \\
\hline & PC & $\mathrm{CC}$ & $\mathbf{P C}$ & $\mathbf{C C}$ & PC & $\mathbf{C C}$ \\
\hline Desvio padrão $\left(\mathrm{m}^{3} / \mathrm{ha}\right)$ & $\pm 26,93$ & $\pm 38,43$ & $\pm 26,67$ & $\pm 22,37$ & $\pm 23,05$ & $\pm 22,23$ \\
\hline Erro padrão $\left(\mathrm{m}^{3} / \mathrm{ha}\right)$ & $\pm 4,26$ & $\pm 10,04$ & $\pm 4,22$ & $\pm 4,35$ & $\pm 3,64$ & $\pm 4,20$ \\
\hline Coeficiente de variação (\%) & 11,35 & 16,58 & 13,89 & 12,04 & 11,97 & 11,96 \\
\hline Erro de amostragem (\%) & $\pm 3,63$ & $\pm 8,76$ & $\pm 4,44$ & $\pm 4,73$ & $\pm 3,83$ & $\pm 4,57$ \\
\hline Intervalo de confiança $\left(\mathrm{m}^{3} / \mathrm{ha}\right)$ & $\pm 8,61$ & $\pm 20,32$ & $\pm 8,53$ & $\pm 8,79$ & $\pm 7,37$ & $\pm 8,50$ \\
\hline
\end{tabular}

De acordo com a tabela 3, para a estimativa do diâmetro médio $(\mathrm{cm})$, o menor erro de amostragem foi obtido para a parcela circular (PC). Yoshida (1991), em reflorestamento de cedro japonês (Cryptomeria japonica D. Don), obteve a seguinte classificação: método de Strand, parcela circular, parcela circular concêntrica e método de Bitterlich. 
Conforme a tabela 3, para a estimativa do número de árvores (N/ha), o menor erro de amostragem foi obtido para o método de amostragem de área fixa com parcela circular (PC). Nakajima et al. (1995, 1996), nas florestas de Shiragadake, Japão, obtiveram a seguinte classificação (quanto à precisão nas estimavas dessas mesmas variáveis): parcela circular, parcela circular concêntrica e método de Strand.

Conforme a tabela 3, para a estimativa de área basal $\left(\mathrm{m}^{2} / \mathrm{ha}\right)$ e volume total $\left(\mathrm{m}^{3} / \mathrm{ha}\right)$, os menores erros de amostragem foram obtidos para o método de amostragem de área fixa com parcela circular (PC), sendo que, para a variável área basal, na condição 2, foi a única vez em que o método de área fixa com conglomerado em cruz foi superior.

Para a área basal, Nakajima et al. (1995, 1996), nas florestas de Shiragadake, Japão, obtiveram a seguinte classificação da precisão: método de Bitterlich, parcela circular concêntrica, método de Strand e parcela circular.

Já para o volume, Mahrer e Vollenweider (1983), em reflorestamentos na Suíça, obtiveram a seguinte classificação: parcela circular concêntrica, método de Strand, método de Bitterlich e parcela circular.

A superioridade da precisão do método de amostragem de área fixa com parcela circular (PC) não se deve ao fato de amostrar $1,7 \%$ mais árvores em média em relação ao conglomerado em cruz (CC), mas, sim, pelo fato de o grau de liberdade utilizado nos cálculos estatísticos do conglomerado em cruz (CC) ser menor, ou seja, para a PC foram utilizados 39 graus de liberdade e para o CC foram utilizados 9 graus.

\section{Classificação da estrutura PC e CC quanto à precisão}

A classificação das estruturas na precisão das estimativas das variáveis é apresentada na tabela 4.

Tabela 4. Classificação das estruturas com base no erro de amostragem.

Table 4. Classification of structures based on error of sampling.

\begin{tabular}{lcc}
\hline Classificação & $\mathbf{1}^{\mathbf{0}}$ Lugar & $\mathbf{2}^{\mathbf{0}}$ Lugar \\
\hline DAP médio $(\mathrm{cm})$ & PC & CC \\
Número de árvores (N/ha) & PC & CC \\
Área basal $\left(\mathrm{m}^{2} / \mathrm{ha}\right)$ & PC & CC \\
Volume total $\left(\mathrm{m}^{3} / \mathrm{ha}\right)$ & PC & CC \\
\hline
\end{tabular}

A tabela 4 mostra que para a estimativa de todas as variáveis correntes avaliadas - diâmetro médio $(\mathrm{cm})$, número de árvores $(\mathrm{N} / \mathrm{ha})$, área basal $\left(\mathrm{m}^{2} / \mathrm{ha}\right)$ e volume total $\left(\mathrm{m}^{3} / \mathrm{ha}\right)$ - a PC foi superior.

\section{Comparação da eficiência relativa entre as estruturas com PC e CC}

Para a análise da eficiência relativa entre as estruturas com parcela circular (PC) e conglomerado em cruz (CC), foram consideradas as três condições simultaneamente.

\section{Classificação das estruturas com $\mathrm{PC}$ e CC quanto à eficiência relativa}

$\mathrm{Na}$ análise de variância (ANOVA) da eficiência relativa para a variável diâmetro médio $(\mathrm{cm}), \mathrm{o}$ teste $\mathrm{F}$ para blocos foi não significativo, ou seja, os blocos não influem na estimativa da variável analisada, não se justificando o controle local feito através dos mesmos. Em relação aos tratamentos, houve diferença significativa ao nível de $5 \%$ de probabilidade, ou seja, os tratamentos não possuem efeitos semelhantes sobre a variável analisada. Sendo assim, dá-se preferência para a estrutura mais eficiente, que é a PC.

Já para as variáveis número de árvores $(\mathrm{N} / \mathrm{ha})$, área basal $\left(\mathrm{m}^{2} / \mathrm{ha}\right)$ e volume total $\left(\mathrm{m}^{3} / \mathrm{ha}\right)$, o teste $\mathrm{F}$ para blocos e tratamentos não foi significativo ao nível de 5\% de probabilidade, ou seja, tanto os blocos quanto os tratamentos possuem efeitos semelhantes sobre as variáveis analisadas. Dessa forma, como são apenas dois tratamentos, dá-se preferência para a estrutura com maior eficiência, que neste caso é a PC.

A tabela 5 mostra que, na estimativa do diâmetro médio $(\mathrm{cm})$, número de árvores $(\mathrm{N} / \mathrm{ha})$, área basal $\left(\mathrm{m}^{2} / \mathrm{ha}\right)$ e volume total $\left(\mathrm{m}^{3} / \mathrm{ha}\right)$, a estrutura com PC foi mais eficiente que o CC.

$\mathrm{Na}$ análise da variável diâmetro médio $(\mathrm{cm})$, conforme a tabela 5 , a diferença média da eficiência 
relativa entre as estruturas é de 1,60 a favor da PC. Isso significa que a PC é 39,7\% mais eficiente em média no levantamento dessa variável, sendo que há diferença estatisticamente significativa entre as eficiências relativas das estruturas.

Tabela 5. Eficiência relativa comparada para as três condições.

Table 5. Relative compared efficiency for the three conditions.

\begin{tabular}{|c|c|c|c|c|c|c|c|c|c|}
\hline \multirow{2}{*}{ Método } & \multirow{2}{*}{$\begin{array}{c}\text { Tempo } \\
\text { (minutos) }\end{array}$} & \multicolumn{2}{|c|}{ DAP (cm) } & \multicolumn{2}{|c|}{ Árvores/ha } & \multicolumn{2}{|c|}{ Área basal (m²/ha) } & \multicolumn{2}{|c|}{ Volume $\left(\mathrm{m}^{3} / \mathrm{ha}\right)$} \\
\hline & & $\mathrm{cv} \%$ & ER & cv\% & ER & cv\% & ER & $\mathrm{cv} \%$ & ER \\
\hline \multicolumn{10}{|c|}{ Condição 1} \\
\hline $\mathrm{PC}$ & 103,36 & 5,34 & 3,39 & 6,01 & 2,67 & 9,60 & 1,05 & 11,35 & 0,75 \\
\hline $\mathrm{CC}$ & 141,33 & 6,17 & 1,86 & 8,32 & 1,02 & 14,47 & 0,34 & 16,58 & 0,26 \\
\hline \multicolumn{10}{|c|}{ Condição 2} \\
\hline $\mathrm{PC}$ & 67,12 & 5,98 & 4,17 & 8,87 & 1,89 & 12,51 & 0,95 & 13,89 & 0,77 \\
\hline $\mathrm{CC}$ & 103,11 & 5,83 & 2,85 & 9,81 & 1,01 & 10,84 & 0,83 & 12,04 & 0,67 \\
\hline \multicolumn{10}{|c|}{ Condição 3} \\
\hline $\mathrm{PC}$ & 69,4 & 5,65 & 4,51 & 5,75 & 4,36 & 11,27 & 1,13 & 11,97 & 1,01 \\
\hline $\mathrm{CC}$ & 111,6 & 5,91 & 2,57 & 10,99 & 0,74 & 11,59 & 0,67 & 11,96 & 0,63 \\
\hline \multicolumn{10}{|c|}{ Média } \\
\hline $\mathrm{PC}$ & 79,96 & 5,66 & 4,02 & 6,88 & 2,97 & 11,13 & 1,04 & 12,40 & 0,84 \\
\hline $\mathrm{CC}$ & 118,68 & 5,97 & 2,43 & 9,71 & 0,92 & 12,30 & 0,61 & 13,53 & 0,52 \\
\hline $\begin{array}{l}\text { Diferença média } \\
\text { (valor absoluto) }\end{array}$ & & & 1,60 & & 2,05 & & 0,43 & & 0,32 \\
\hline
\end{tabular}

Já na análise do número de árvores (N/ha), área basal $\left(\mathrm{m}^{2} / \mathrm{ha}\right)$ e volume total $\left(\mathrm{m}^{3} / \mathrm{ha}\right)$, conforme a tabela 5, a diferença média da eficiência relativa entre as estruturas foi de 2,05, 0,43 e 0,32 em favor da PC. Isso significa que a PC é $68,9 \%, 41,2 \%$ e $38,3 \%$ mais eficiente, em média, no levantamento dessas variáveis, sendo que não há diferença estatisticamente significativa entre as eficiências relativas das estruturas.

\section{CONCLUSÃO}

- Para todas as variáveis - diâmetro médio $(\mathrm{cm})$, número de árvores $(\mathrm{N} / \mathrm{ha})$, área basal $\left(\mathrm{m}^{2} / \mathrm{ha}\right)$ e volume total $\left(\mathrm{m}^{3} / \mathrm{ha}\right)$-, indica-se a utilização do método de área fixa com parcela circular (PC), por ser mais preciso e eficiente no levantamento dessas variáveis, nestas condições.

\section{REFERÊNCIAS}

AVERY, T. E.; BURKHART, H. Forest measurements. New York, McGraw-Hill Book Company, 1983. $331 \mathrm{p}$.

BOGNOLA, I.; POTTER, R. O.; CARVALHO, A. P.; FASOLO, P. J.; BHERING, S. B.; MARTORANO, L. G. Caracterização dos solos do município de Carambeí, PR. Boletim de Pesquisa e Desenvolvimento, Rio de Janeiro, p. 75, 2002.

DRUSZCZ, J. P. Comparação do método de Bitterlich com três variações estruturais do método de área fixa com unidades circulares em plantio de Pinus taeda L. 120 f. Dissertação (Mestrado em Manejo Florestal) - Universidade Federal do Paraná. Curitiba, 2008.

DRUSZCZ, J. P.; NAKAJIMA, Y. N.; PÉLLICO NETTO, S.; YOSHTANI JÚNIOR, M. Comparação entre os métodos de amostragem de Bitterlich e de área fixa com parcela circular em plantação de Pinus taeda. Revista Floresta, Curitiba, v. 40, n. 4, p. 739 - 754, 2010. 
DRUSZCZ, J. P.; NAKAJIMA, Y. N.; PÉLLICO NETTO, S.; MACHADO, S. A. Custos de inventário florestal com amostragem de Bitterlich (PNA) e conglomerado em cruz (CC) em plantação de Pinus taeda L. Revista Scientia Forestalis, Piracicaba, v. 40, n. 94, p. 231 - 239, 2012.

DRUSZCZ, J. P.; NAKAJIMA, Y. N.; PÉLLICO NETTO, S.; MACHADO, S. A.; MELLO, A. A.; CAMPOS, A. P. G. Eficiência de inventário florestal com amostragem ponto de Bitterlich e conglomerado em linha em plantação de Pinus taeda. Revista Floresta, Curitiba, v. 42, n. 3, p. 527 - 538, 2012.

FREESE, F. Elementary forest sampling. Washington: Forest Service, Agriculture Handbook n. 232, 1962, $91 \mathrm{p}$.

MAHRER, F.; VOLLENWEIDER, C. National forest inventory. Swiss: Swiss Federal Institute of Forestry Research, 1983, 26 p.

MAURICIO, J.; VINCENT, L.; MORET, A. Y.; QUEVEDO, A. Comparación entre modalidades de muestreo em plantaciones de Pino Caribe en el oriente de Venezuela. Revista Forestal Venezolana, Mérida, v. 49, n. 1, p. 17 - 25, 2005.

NAKAJIMA, Y. N.; PÉLLICO NETTO, S.; DRUSZCZ, J. P.; YOSHTANI JÚNIOR, M. Comparação da precisão entre os métodos de amostragem linha e parcela circular concêntrica em povoamentos de Pinus elliottii. Revista Acadêmica: Ciências Agrárias e Ambientais, Curitiba, v. 9, n. 2, p. 131 - 139, 2011.

NAKAJIMA, N. Y.; YOSHIDA, S.; IMANAGA, M. Comparison among four ground-survey methods as a continuous forest inventory system for forest management. J. Jpn. For. Soc., Kagoshima, v. 77, n. 6, p. 573 - 580, 1995.

. Comparison of the accuracies of four ground-survey methods used for estimating forest stand values on two occasions. J. For. Plann., Kagoshima, p. 137 - 144, 1996.

NAKAJIMA, N. Y. Comparison of four ground-survey methods when used as permanent samples in the continuous forest inventory for forest management. $91 \mathrm{p}$. Tese (Doutorado em Engenharia Florestal) - The United Graduate School of Agricultural Sciences, Kagoshima University. Kagoshima, 1997.

NAKAJIMA, N. Y.; KIRCHNER, F. F.; SANQUETTA, C. R.; POSONSKI, M. Elaboração de um sistema de amostragem para estimativa de valores correntes e mudança/crescimento em reflorestamento de pinus. Curitiba: CNPq/UFPR, 1998. 33 p. (CNPq - Pesquisa na modalidade recémdoutor). Projeto concluído.

NISHIZAWA, M. Forest mensuration. Tokyo: Nourinshuppan, 1972.

PÉLLICO NETTO, S.; BRENA, D. Inventário florestal. Curitiba: Universidade Federal do Paraná, 1997. $316 \mathrm{p}$.

QUEIROZ, W. T. Técnicas de amostragem em inventário florestal nos trópicos. Belém. 1998. 147 p.

SANQUETTA, C. R.; WATZLAWICK, L. F.; DALlA CÔRTE, A.; FERNANDES, L. A. V. Inventários florestais: planejamento e execução. 2. ed. Curitiba, 2009, 271 p.

SPARKS, J.; MASTERS, R.; PAYTON, M. Comparative evaluation of accuracy and efficiency of six forest sampling methods. Proc. Okla. Acad. Sci., Oklahoma, p. 49 - 56, 2002.

YOSHIDA, S. Studies on the continuous forest inventory system: comparison of survey methods on the ground. The Bulletin of the Faculty of Agriculture, Kagoshima University 41, p. 7 - 12, 1991. 